\title{
New Navigation Strategy for G-Bot
}

\author{
Nyayu Latifah Husni ${ }^{1, *}$ Qodri Maulana ${ }^{1}$ Ekawati Prihatini ${ }^{1}$ Ade Silvia \\ Handayani $^{1}$ Firdaus $^{2}$ Masayu Anisah ${ }^{1}$ \\ ${ }^{1}$ State Polytechnic of Sriwijaya, Electrical Engineering, Palembang, Indonesia \\ ${ }^{2}$ Sriwijaya University, Computer Science Department, Palembang, Indonesia \\ ${ }^{*}$ Corresponding author. Email: nyayu_latifah@polsri.ac.id
}

\begin{abstract}
This paper discusses about the navigation of G-Bot is (Garbage Robot). The G-Bot is designed to attract the human intention (especially kids) to throw the garbage in proper place. The G-Bot in this research has an attracted and colorful body. It can be plugged and assembled easily so that it can be washed in order to keep its cleanliness. However, how it navigates to the target and avoids the obstacles still become the problems. In this research, a new navigation strategy using fuzzy logic is implemented to the G-Bot in order to help it to keep its navigation to the correct path. The navigation of G-Bot is conducted in the aisle of the classrooms using 3 obstacles. It is tested to navigate from room 1 to room 2 and vice versa. From the experiment, it can be concluded that the G-Bot has been successful to reach the target (room 1 and also room 2) in about 21 minutes.
\end{abstract}

Keywords: Navigation, Fuzzy Logic, G-Bot

\section{INTRODUCTION}

Garbage is residual material that is not used or unwanted after the use. Garbage is divided into several types. When viewed from its nature, garbage is classified into organic and inorganic garbage. The organic one is the garbage that can be easily decomposed (can be broken down), such as food scraps, vegetables, dry leaves, and others. This garbage can be further processed into compost. Meanwhile, inorganic one is the garbage that cannot be easily to be decomposed, such as plastic food wrapping containers, plastic toys, drinking bottles, glasses, cans, wood, and others.

In Indonesia, the garbage has been being a very serious problem that affects not only the social part but also the economic and the culture [1]-[12]. Almost all cities in Indonesia has problems in managing garbage. The lack of public concern for the surrounding environment and the lack of garbage bins are some of the factors that increase garbage accumulation in Indonesia. Various efforts have been made to overcome those problems, including making regulations and sanctions for violators, educating people about the importance of disposing of garbage in a proper place, sorting and managing garbage, and developing garbage applications. However, these efforts have not been maximized. It is due to only a few communities want to be involved in this problem.
The importance of educating children about the dangers of disposing the garbage inappropriately from an early age will greatly help to increase their care for the environment. This will have an impact on the habits of society in the future. In the current era of digitalization, smart educational games for sorting garbage can be a teaching medium in instilling a culture of disposing of garbage in its place. However, the game is only a simulation and there are many negative impacts that it can cause.

Based on the problems described above, this study offers a garbage overcoming concept using a garbage robot (G-Bot). Besides being useful as a garbage collector, this G-Bot is also very effective in attracting children's attention to build positive energy, i.e., by accustoming them to throwing garbage in the right place.

Many garbage robots are developed with very expensive selling prices, therefore, only few people can use them. In this study, a low cost garbage robot was built by utilizing recycled materials, especially for the robot casings and frames. It used newspaper molded plates which can be obtained at low prices. Besides the high selling price, garbage robots on the market also cannot be washed. This is because the electronic components are attached directly to the body of the robot. The garbage robot offered in this study consisting of 3 parts that can be assembled.

Another advantage of using a garbage robot as a garbage carrier is that it can move automatically, 
therefore, it can save energy and time for people who will throw garbage. However, how to control the garbage robot, so that the robot can walk and work as it should be, is a challenge for researchers. Therefore, the researcher proposes a concept of motion control for GBot based on Artificial Intelligence.

In this research, a fuzzy logic is applied to the GBot in order to maintain the G-Bot motion. This fuzzy logic helps the G-Bot to navigate to the correct path. There are a lot of researchers who has used fuzzy logic in the research, such as in [13]-[20].

\section{PROPOSED METHOD}

In this study, the G-Bot is accomplished with two active wheels (rubber AGV 120 mm, hole custom 6-15 $\mathrm{mm}$, and Load $\max 10 \mathrm{~kg}$ ) and one free wheel for its maneuvering. Based on the Figure 1, the parameters of the $X$ and $Y$ axes are the positions of the robot. $C$ is the center of the robot. $\theta$ is the angle formed by the $X$-axis, and $\omega$ is the angular velocity. The distance between the wheel centers is shown in $L$. The linear velocity for each wheel is symbolized as $V 1$ and $V 2$. Linear velocity centered on $C$ is shown in $V$ and $V n$. Its angular speed is affected by the $M 1$ and $M 2$ motors respectively.

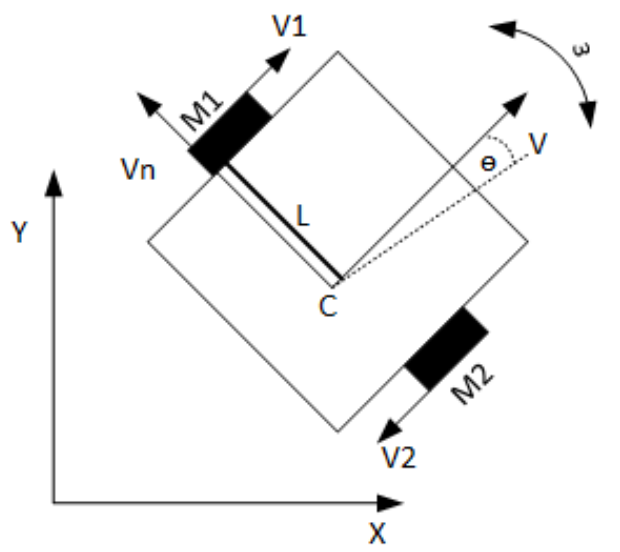

Figure 1 Illustration of the direction of movement

The basic formulation for kinematic in the robot is shown in Equation (1). The $T_{N H}(q)$ parameter is a nonholonomic transformation of the mobile robot. The $u(t)$ is input used to estimate the position and velocity of the robot in cartesian space.

$$
\left[\begin{array}{l}
X(t) \\
Y(t) \\
\theta(t)
\end{array}\right]=T_{N H}(q) \cdot u(t)
$$

In Figure 1, the linear velocity for each of $V 1$ and $V 2$ can be expressed using Equation (2)

$$
\begin{aligned}
& V 1(t)=V n(t)+L . \omega(t) \\
& V 2(t)=-V(t)+L . \omega(t)
\end{aligned}
$$

If the velocity of $V 1$ and $V 2$ in Equation (2) are added together, it will produce an angle that is shown in Equation (3).

$$
\begin{gathered}
\theta(t)=\omega(t)=\frac{1}{2 L}(V 1(t)+V 2(t)) \\
V=\frac{1}{2}(V 1(t)-V 2(t))
\end{gathered}
$$

Equation (4) shows the actual position obtained

$$
\begin{gathered}
X(t)=V(t) \cos \theta(t)-V n(t) \cos (90-\theta)(t)) \\
Y(t)=V(t) \sin (90-\theta)(t)+V n(t) \sin \theta(t) \\
\theta(t)=\omega(t)
\end{gathered}
$$

The formation of non-holonomic constrains in the robot direction is shown in Equation (5).

As mentioned before, the designing of the Smart GBot in this research uses the fuzzy logic method in order to control the robot. In the application of this fuzzy logic, there are several steps that should be conducted in order to manage the sensor data input before the output signal is finally generated to the actuator (See Figure 2). The processes are first begun by the fuzzification, then it is continued with the inference engine and the defuzzification process.

The G-Bot smart navigation system is designed to regulate the robot's motion to reach its destination. The navigation system is designed so that the robot recognizes the position and direction of the destination based on the data provided. Therefore, to make it easier read the navigation, the first thing that should be done in software design is to make the flowchart. With the flowchart, the direction of the robot's navigation movement can be understood. In this research, the flowchart is shown in Figure 3.

$$
\left[\begin{array}{l}
X(t) \\
Y(t) \\
\theta(t)
\end{array}\right]=\left[\begin{array}{cccc}
-\frac{1}{2} \cos (90-\theta)(t) & -\frac{1}{2} \cos \theta(t) & \frac{1}{2} \cos (90-\theta)(t) & \frac{1}{2} \cos \theta(t) \\
\frac{1}{2} \sin (90-\theta)(t) & -\frac{1}{2} \sin \theta(t) & -\frac{1}{2} \sin (90-\theta)(t) & \frac{1}{2} \sin \theta(t) \\
\frac{1}{2 L} & \frac{1}{2 L} & \frac{1}{2 L} & \frac{1}{2 L}
\end{array}\right]\left[\begin{array}{l}
V 1(t) \\
V 2(t)
\end{array}\right]
$$




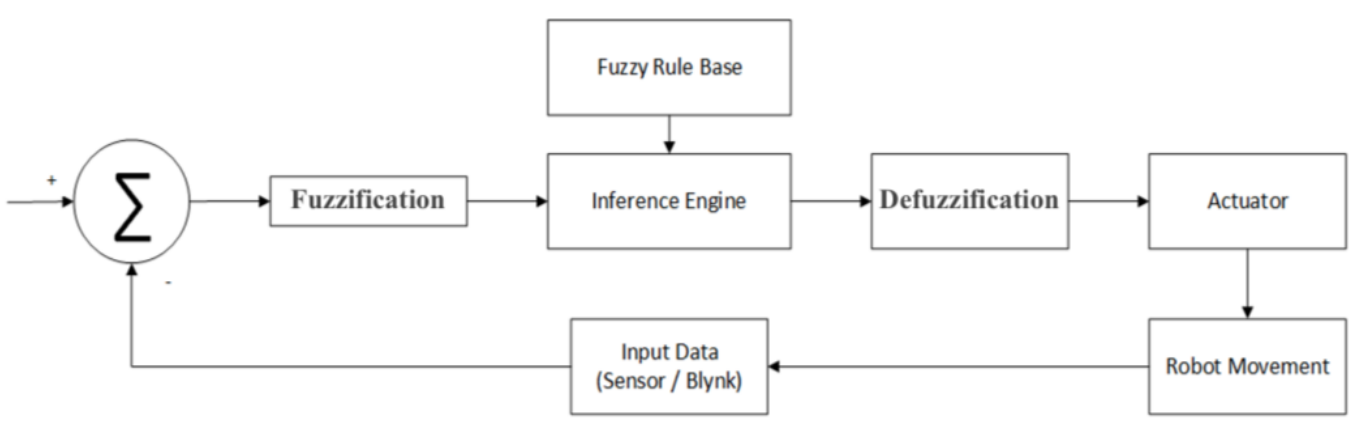

Figure 2 Fuzzy logic control mechanism

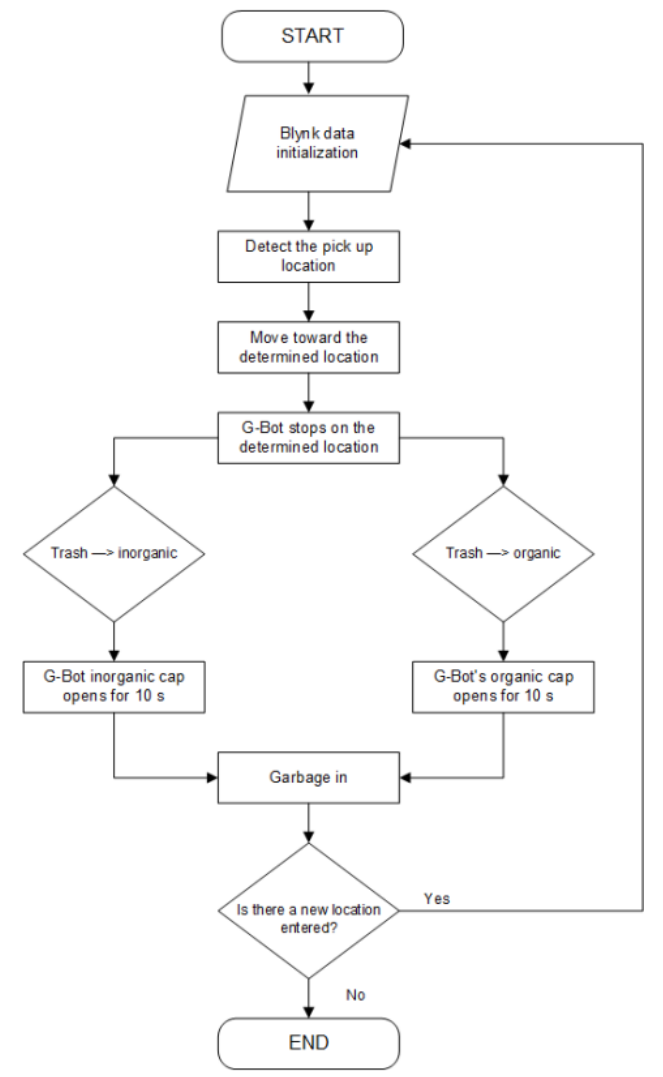

Figure 3 Flowchart Smart G-Bot

\subsection{Fuzzification}

Fuzzification is the process of transforming crisp data into linguistic variables (fuzzy sets), with a certain degree of membership or what can be called as the input fuzzy value. In this process, the resulting fuzzy input value is influenced by the input membership design that is used.

In this research, the input membership function is designed using 3 inputs. The navigation system is based on those 3 inputs, namely Left Ultrasonic (S1), Front Ultrasonic (S2) and Right Ultrasonic (S3).

$\begin{array}{llll}\text { Near }(\mathrm{N}) \quad \mathrm{x}<35 & = & 1 \\ & 35<\mathrm{x} \leq 50 & = & \frac{x-50}{35-50} \\ & \mathrm{x}>50 & = & 0 \\ \text { Far }(\mathrm{F}) & \mathrm{x}<35 & = & 0 \\ & 35<\mathrm{x} \leq 50 & = & \frac{x-35}{50-35} \\ & 50<\mathrm{x} \leq 65 & = & \frac{x-65}{50-65} \\ & \mathrm{x}>65 & = & 0 \\ \text { Very Far } \quad \mathrm{x}<50 & = & 0 \\ (\text { VF) } & 50<\mathrm{x} \leq 65 & = & \frac{x-50}{65-50} \\ & \mathrm{x}>65 & = & 1\end{array}$

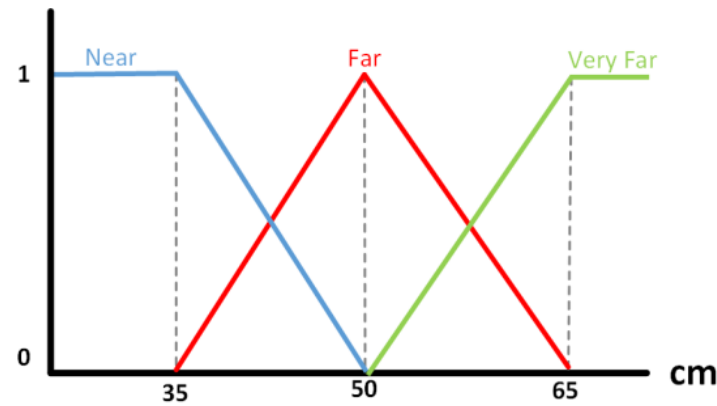

Figure 4 Ultrasonic fuzzy input

From these inputs, the membership function is created with different linguistic variable values. The three inputs have 3 linguistic values, namely Very Far (VF), Far (F), and Near (N). The value of the degree of membership of each linguistic value is 1 . The equation for the fuzzy logic membership function based on the ultrasonic sensor distance parameter is shown in Equation (6).

Based on the process of calculating the fuzzification of the ultrasonic sensor distance, the fuzzy logic inputs with their membership function graph can be seen in Figure 4. 


\subsection{Rule Evaluation (Inference Engine)}

Rule evaluation is a decision-making process (inference) based on the rules set on the basis of a rule, i.e., to connect fuzzy input with fuzzy output. These rules take the form of (IF ... Then).

Table 1 Fuzzy control rules

\begin{tabular}{ccccc}
\hline & INPUT & \multicolumn{2}{c}{ OUTPUT } \\
\hline S1 & S2 & S3 & M1 & M2 \\
\hline VF & VF & VF & S & S \\
\hline VF & VF & F & S & M \\
\hline VF & VF & N & S & F \\
\hline VF & F & VF & M & M \\
VF & F & F & S & M \\
\hline VF & F & N & S & F \\
\hline VF & N & VF & F & F \\
VF & N & F & F & F \\
\hline VF & N & N & S & F \\
\hline F & VF & VF & M & S \\
\hline F & VF & F & M & S \\
\hline F & VF & N & F & S \\
\hline F & F & VF & M & S \\
\hline F & F & F & M & M \\
\hline F & F & N & M & F \\
\hline F & N & VF & M & M \\
\hline F & N & F & M & M \\
\hline F & N & N & M & F \\
\hline N & VF & VF & F & S \\
\hline $\mathbf{N}$ & VF & F & F & S \\
\hline $\mathbf{N}$ & VF & N & F & S \\
\hline $\mathbf{N}$ & F & VF & F & S \\
\hline $\mathbf{N}$ & F & F & F & M \\
\hline $\mathbf{N}$ & F & N & F & M \\
\hline $\mathbf{N}$ & N & VF & F & S \\
\hline $\mathbf{N}$ & N & F & F & M \\
\hline $\mathbf{N}$ & N & N & F & F \\
\hline & & & & \\
\hline
\end{tabular}

Note:

Input Parameters:

$$
\begin{array}{ll}
\mathrm{N} & : \text { Near } \\
\mathrm{F} & : \text { Far } \\
\mathrm{VF} & \text { : Very Far }
\end{array}
$$

Output Parameters:

$$
\begin{array}{ll}
\mathrm{F} & : \text { Fast } \\
\mathrm{M} & : \text { Medium } \\
\mathrm{S} & \text { : Slow }
\end{array}
$$

There are 2 Inference Engine processes: a. Aggregation: the process of calculating the "IF"; and b. Composition: The calculation process on "Then".

The defined rules are used to specify the relationship between the input fuzzy set and the output fuzzy set. This robot has two navigation modes, namely follow right and follow left. To follow the right the robot will explore the room in a clockwise direction. On the contrary, to follow the left the robot will explore the room in counterclockwise direction. To right follow, the robot only uses the right and front sensor input. Meanwhile, to left follow, the robot only used the front and left sensor input. Table 1 shows the design of control rules in the navigation of this smart G-Bot.

\subsection{Defuzzification}

Defuzzification is the process of finding the value of linguistic variables based on the degree of membership they have. The defuzzification process produces the desired output from the previous process. The defuzzification process has an output membership function in the form of a vertical line (singleton) as represented in Equation (7).

\section{Defuzzification*}

$$
Z=\frac{\omega 1 \cdot \alpha 1+\omega 2 \cdot \alpha 2+\cdots+\omega n \cdot \alpha n}{\varepsilon \alpha}
$$

where

$\mathrm{Z}=$ Defuzzification

$\omega=$ PWM motor

$\alpha=$ Fuzzy Rules Value

\subsection{Circuit Schematic}

Electronic circuit design is the process of making circuits, PCB layout and printing and wiring (wiring diagrams). The creation of a series and layout in this research uses the eagle application and Corel Draw X7. As can be seen in Figure 5, the schematic design is consisted of some components, such as: i) 1 Arduino Mega 2560 rev 3 which functions as the minimum system used; ii) 5 ultrasonic sensors, 3 of which serve as railroad navigation readings and the other 2 for reading garbage height; iii) 1 colorr sensor TCS3200 that has function as a colorr detector when the robot is moving; iv) 1 Compass sensor functions as a robot angle adjustment in the initial position; v) 1 DHT11 sensor which functions as a humidity and temperature detector; vi) 1 MQ-7 sensor as a gas detector; vii) 1 piece Motor Driver Cytron MDD10A Rev.02; viii) 2 PG45 motors, function as robot driving motors; ix) 2 MG996 Servo Motors, function as control for opening and closing the robot's; $x$ ) 1 I2C and $120 x 4$ LCD as screen media to display the temperature, garbage capacity, and robot positions. 


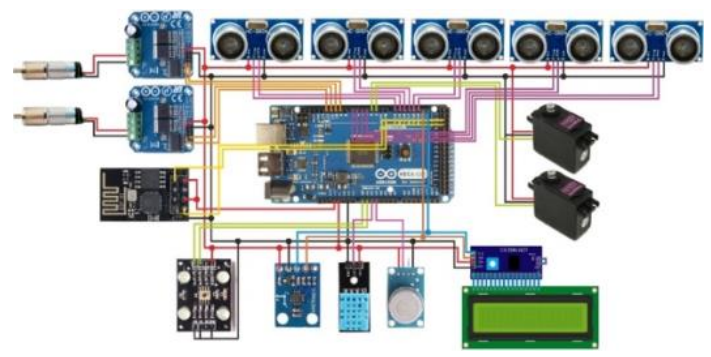

Figure 5 Smart G-Bot Schematic Diagram

\subsection{Mechanical Design}

Hardware design includes mechanical and electrical design stages. In designing the Smart G-Bot, there are several pieces of hardware that are used to complement the equipment of this robot. The full design can be seen in Figure 6.

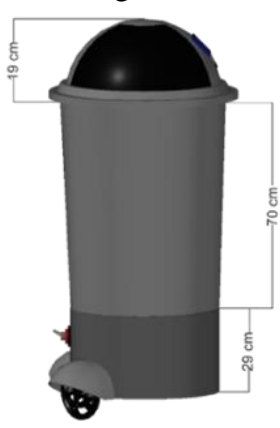

(a)

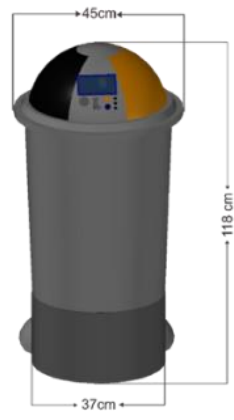

(b)
Figure 6 (a) Side view, (b) Front view

\section{EXPERIMENTAL RESULT}

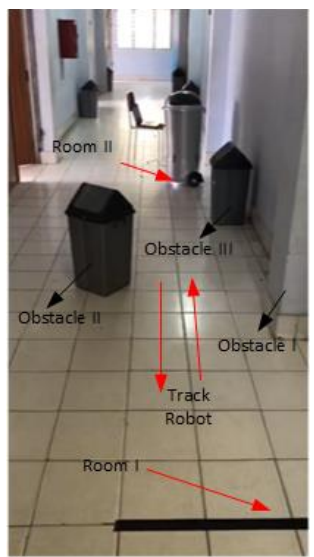

Figure 7 Experimental arena.

The navigation of the G-Bot to the target was tested in the isle of Electrical Engineering of State Polytechnic of Sriwijaya.
The G-Bot should be able to navigate from ROOM 1 to ROOM 2 and vise versa smoothly. 3 of obstacles were placed in the experimental arena, i.e. 2 garbage boxes that were placed arbitrarily and 1 obstacle of the room pole (as shown in Figure 7)

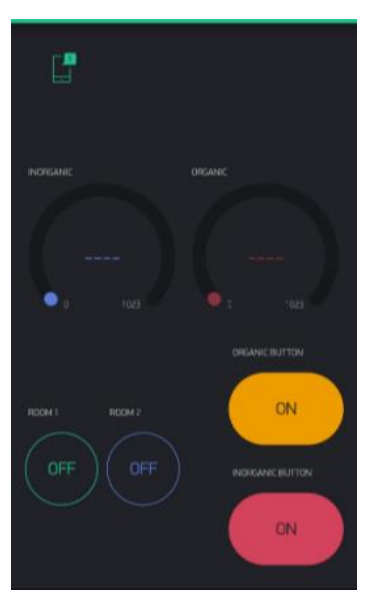

(a) Blynk Display

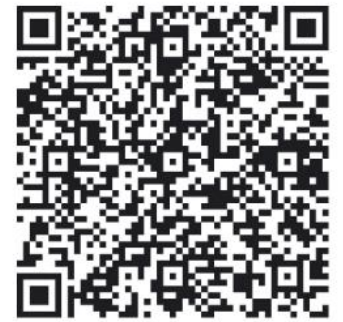

To start using The Blynk:

1. Download Blynk App: http://j.mp/blynk_Android or http://j.mp/blynk_iOS

2. Touch the QR-code icon and point the camera to the code

3. Enjoy the application (b) G-Bot Barcode
Figure 8. G-Bot Controller

Figure 8 shows the G-Bot controller. This controller is designed using Blynk Application. The display of the controller is useful for controlling and monitoring the G-Bot. In Figure 8, it can be seen that there are some features, such as: the monitoring display of inorganic and organic garbage capacity, the button for opening the G-Bot's cover, and the button of moving the G-Bot to ROOM 1 or ROOM 2.

\subsection{Case 1}

In Case 1, The G-Bot was designed to move from ROOM 2 to ROOM 1. The initial position of the GBot is shown in Figure 9 (a). When the push button "ROOM 1" in the Blynk application was pressed as shown in Figure 9 (b), the G-Bot then started to navigate. At $t=1 \mathrm{~s}$, as shown in Figure 9 (c), the GBot was so closed with the garbage box that functioned as its first obstacle, however, its distance sensors did not consider it was a significant obstacle to be hint, therefore it just kept moving left forward with different left and right PWM, i.e. $100 \mathrm{rpm}$ for the left motor and $175 \mathrm{rpm}$ for the right motor. Due to the right PWM is higher than the left one, the G-Bot moved to the left as shown in Figure 9 (d). At Figure 9 (e), the G-Bot detected the second obstacle on it right side. 


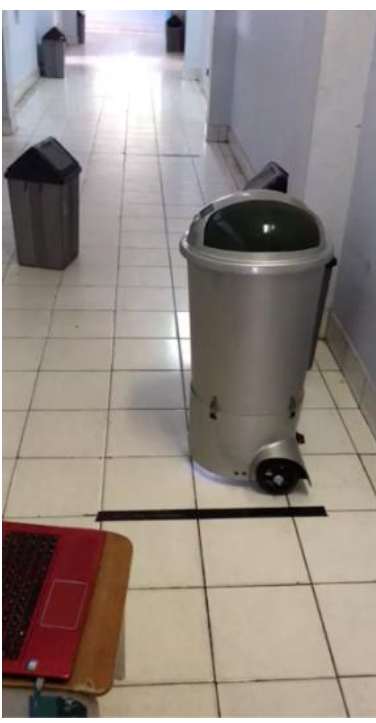

(a) Initial Position

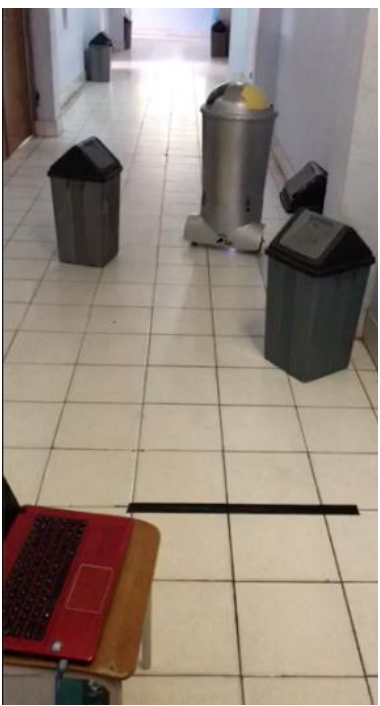

(e) $t=9 \mathrm{~s}$

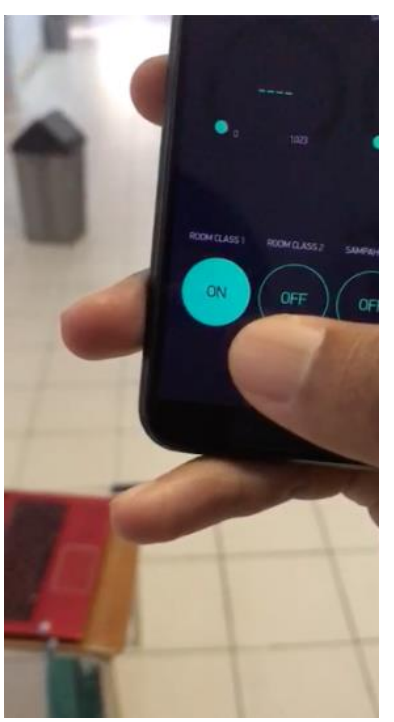

(b) ROOM 1 button ON

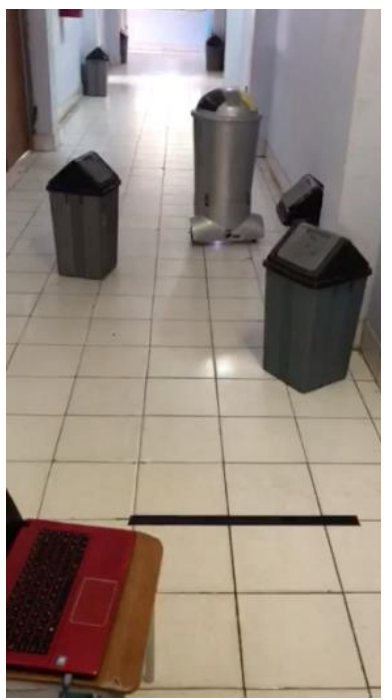

(f) $\mathrm{t}=10 \mathrm{~s}$

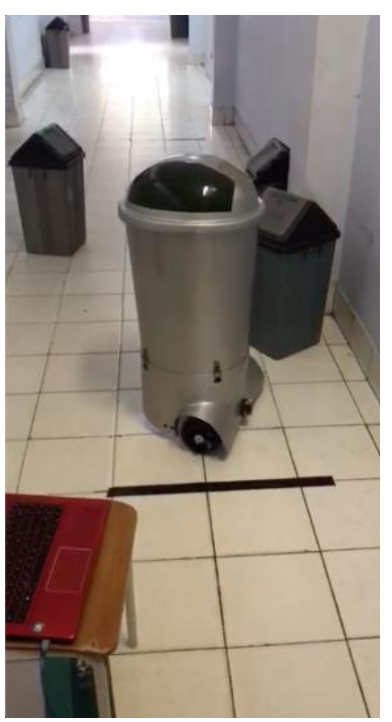

(c) $\mathrm{t}=1 \mathrm{~s}$

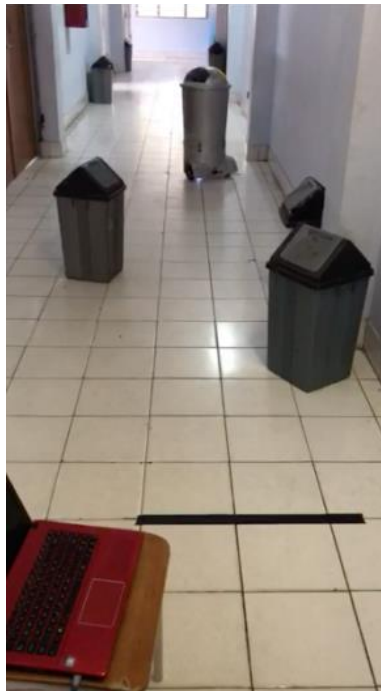

(g) $\mathrm{t}=19 \mathrm{~s}$

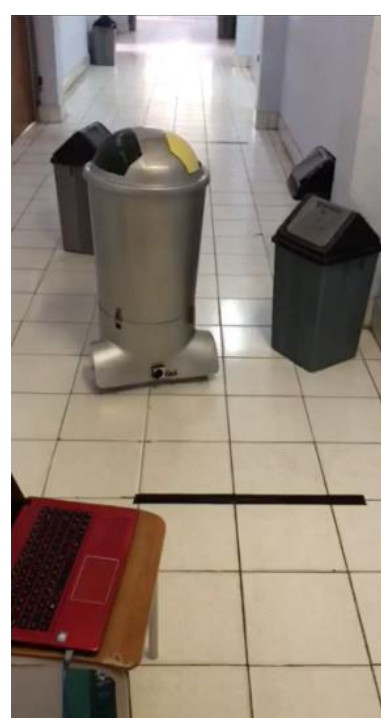

(d) $t=4 s$

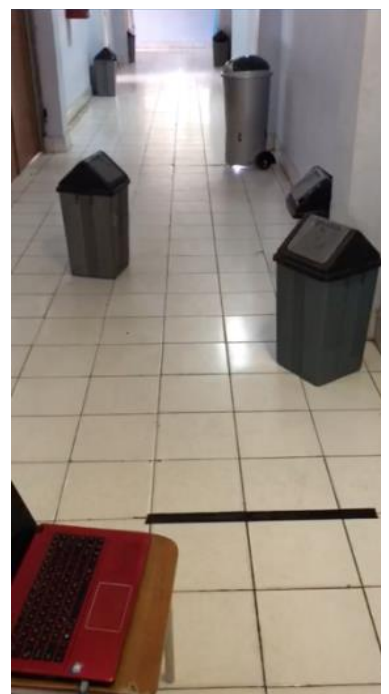

(h) $\mathrm{t}=21 \mathrm{~s}$

Figure 9 Navigation of G-Bot in Case 1

It then changed its navigation by renewing its left and right PWM, i.e. $146 \mathrm{rpm}$ for the right motor and 99 for the left motor. Thus, the robot moved quite left as shown in Figure 9 (f). The G-Bot moved forward smoothly at $\mathrm{t}=11 \mathrm{~s}$ until $\mathrm{t}=18 \mathrm{~s}$. At $\mathrm{t}=19 \mathrm{~s}$, the $\mathrm{G}-$ Bot has passed the black line that became the set point of the target, it then controlled its moving to be its initial position (Figure $9(\mathrm{~g})$ ). At $\mathrm{t}=21 \mathrm{~s}$ (Figure 9 (h)), the G-Bot has reached its final position.

\subsubsection{Fuzzification for Case 1}

Table 2 shows the data of the G-Bot while moving from ROOM 2 to ROOM 1. The data of 3 ultrasonic sensors, the data of the left and the right PWM were recorded in that. table.

Table 2 Experimental data of Case 1

\begin{tabular}{|c|c|c|c|c|c|}
\hline \multirow{2}{*}{$\begin{array}{c}\text { Time } \\
(\mathrm{s})\end{array}$} & \multicolumn{3}{|c|}{ Input } & \multicolumn{2}{c|}{ Output } \\
\cline { 2 - 6 } & \multicolumn{2}{|c|}{$\begin{array}{c}\text { Ultrasonic Sensor } \\
\text { Distance (cm) }\end{array}$} & \multicolumn{2}{c|}{ PWM } \\
\cline { 2 - 6 } & S1 & S2 & S3 & left & Right \\
\hline 1 & 34 & 68 & 38 & 200 & 99 \\
\hline 4 & 72 & 87 & 43 & 100 & 175 \\
\hline 9 & 85 & 75 & 51 & 99 & 146 \\
\hline 10 & 70 & 84 & 37 & 99 & 180 \\
\hline 19 & 38 & 72 & 70 & 189 & 100 \\
\hline 20 & 75 & 34 & 38 & 120 & 198 \\
\hline
\end{tabular}


For calculating the fuzzy value, an example of experiment in the 4 second measurement of the Case 1 is taken (See Table 2). The fuzzy calculation process goes through several stages, as follows:

From the setting data, it can be seen that the data of each membership functions in fuzzy input an be calculated using Equation (6). From the experiments shown in Table 2, it can be concluded that ultrasonic sensor distance parameters are as follows: $\mathrm{S} 1=72$, S2 $=87$ and $\mathrm{S} 3=43$.

Therefore:

Ultrasonic Sensor Distance 72 (S1)

$\begin{array}{ll}\mathrm{N} & \mathrm{x}>50=0 \\ \mathrm{~F} & \mathrm{x}>65=0 \\ \mathrm{VF} & \mathrm{x}>65=1\end{array}$

Ultrasonic Sensor Distance 87 (S2)

$$
\begin{array}{ll}
N & x>50=0 \\
F & x>65=0 \\
\text { VF } & x>65=1
\end{array}
$$

Ultrasonic Sensor Distance 43 (S3)

$$
\begin{array}{lll}
\mathrm{N} & 35<\mathrm{x} \leq 50 & =\frac{x-35}{35-50}=\frac{43-50}{35-50}=0.46 \\
\mathrm{~F} & 35<\mathrm{x} \leq 50 & =\frac{x-35}{50-35}=\frac{43-35}{50-35}=0.53 \\
\mathrm{VF} & \mathrm{x}<50 & =0
\end{array}
$$

Based on the above calculations, the equation is:

\section{VF VF N \\ 2. VF VF F}

See Table 3 for the Detail of the ultrasonic distance calculation value.

Table 3 Ultrasonic Distance Sensor Calculation Value

\begin{tabular}{|c|c|c|c|c|c|}
\hline \multirow{2}{*}{ No. } & \multicolumn{2}{|c|}{ Ultrasonic Distance Calculation Value } & \multicolumn{2}{c|}{ PWM } \\
\cline { 2 - 6 } & S1 & S2 & S3 & Left & Right \\
\hline \multirow{2}{*}{1} & VF & VF & N & S & \multirow{2}{*}{ F } \\
\hline \multirow{2}{*}{2} & 1 & 1 & 0.46 & & \multirow{2}{*}{ M } \\
\hline
\end{tabular}

Table 4 Results of Ultrasonic Sensor Distance Defuzzification Calculations

\begin{tabular}{|c|c|c|c|c|}
\hline $\begin{array}{c}\text { Least Value Fuzzy } \\
\text { Rule }(\alpha)\end{array}$ & $\begin{array}{c}\text { Left PWM } \\
(\omega L \text { eft })\end{array}$ & $\begin{array}{c}\text { Right PWM } \\
(\omega \text { Right })\end{array}$ & $w \alpha$ Left & w. $\alpha$ Right \\
\hline 0.46 & 200 & 100 & 46 & 92 \\
\hline 0.53 & 150 & 100 & 53 & 79.5 \\
\hline$\Sigma \alpha=0.99$ & \multicolumn{2}{|c|}{ Total : } & 99 & 171.5 \\
\hline
\end{tabular}

\subsubsection{Defuzzification for Case 1}

Table 4 shows the calculation of the ultrasonic sensor distance defuzzification. This data was obtained by using the Equation (7).

In this research, the PWM values (output) are given in Equation (8).

$$
\begin{aligned}
& \mathrm{F}=200 \text { Fast } \\
& \mathrm{M}=150 \text { Medium } \\
& \mathrm{S}=100 \text { Slow }
\end{aligned}
$$

Therefore:

$$
\text { ZRight }=(w . \alpha \text { Right }) / \Sigma \alpha=171.5 / 0.99=173.2
$$

To test the result, compare the measurement and calculation value. The error of the left and right motor PWM can be calculated, as follows:

error $=\left|\frac{(\text { calculation value }- \text { measurement value })}{\text { calculation value }}\right| \times 100 \%$

\section{Left Motor:}

$$
\begin{aligned}
& \text { error }=\left|\frac{100-100}{100}\right| \times 100 \% \\
& \text { error }=\left|\frac{0}{100}\right| \times 100 \%
\end{aligned}
$$


error $=0 \%$

\section{Right Motor:}

error $=\left|\frac{173.2-175}{173.2}\right| \times 100 \%$

error $=\left|\frac{1.8}{173.2}\right| \times 100 \%$

error $=1.04 \%$

Thus, the output of the fuzzy logic (defuzzification) of the ultrasonic sensor distance is 173.2 (Right PWM) and 100 (Left PWM) with an error of $1.04 \%$ for the right motor and $0 \%$ for the left motor. This calculation is only the sampling. To find all of the calculation value, the steps that has been presented before can be adopted.

\subsection{Case 2}

In Case 2, The G-Bot was designed to move from ROOM 1 to ROOM 2. In this condition, when the push button "ROOM 2" in the Blynk application was pressed (Figure 10 (a)), the G-Bot then started to change its position from its initial to the target. At $\mathrm{t}=$ $3 \mathrm{~s}$, as shown in Figure 10 (b), the G-Bot met the garbage box as its first obstacle, the G-Bot then changed its direction by making improvement on its left and right PWM, i.e. $195 \mathrm{rpm}$ for the right motor and $100 \mathrm{rpm}$ for the left motor. Due to the left PWM is smaller than the right one, the G-Bot moved to the left. However, due to it detected the obstacles, it tried to hint it and started to move to the right to get the position as shown in Figure 10 (c). In figure 10 (c), the robot could run smoothly (at $t=4 \mathrm{~s}$ ). It continued until $\mathrm{t}=13 \mathrm{~s}$. Although it met the second obstacles on the $\mathrm{t}=7 \mathrm{~s}$, however due to the obstacles did not limit its trajectory, in addition, its fuzzy logic could work well, therefore, it only kept moving forward until $\mathrm{t}=$ $13 \mathrm{~s}$, as shown in Figure 10 (d) until Figure 10 (f). At $\mathrm{t}=14 \mathrm{~s}$, the G-Bot has passed the black line that became the set point of the target, it then controlled its moving to be its initial position (Figure $10(\mathrm{~g})$ ). At $\mathrm{t}=$ $16 \mathrm{~s}$ (Figure $10(\mathrm{~h})$ ), the G-Bot kept on backward to reach its final position. This movement continued until it could reach the correct position. The robot could accomplish the task in 17 seconds

Table 5 Experimental Data of Case 2

\begin{tabular}{|c|c|c|c|c|c|}
\hline \multirow{2}{*}{$\begin{array}{c}\text { Time } \\
(s)\end{array}$} & \multicolumn{3}{|c|}{ Input } & \multicolumn{2}{c|}{ Output } \\
\cline { 2 - 6 } & \multicolumn{2}{|c|}{$\begin{array}{c}\text { Ultrasonic Sensor } \\
\text { Distance (cm) }\end{array}$} & \multicolumn{2}{c|}{ PWM } \\
\cline { 2 - 6 } & S1 & S2 & S3 & Left & Right \\
\hline 3 & 36 & 93 & 83 & 100 & 195 \\
\hline 4 & 49 & 62 & 81 & 153 & 99 \\
\hline 7 & 42 & 66 & 74 & 179 & 97 \\
\hline 9 & 41 & 68 & 87 & 180 & 100 \\
\hline 13 & 38 & 81 & 77 & 175 & 176 \\
\hline 14 & 49 & 69 & 81 & 152 & 100 \\
\hline 16 & 60 & 87 & 68 & 116 & 98 \\
\hline
\end{tabular}

Table 5 shows the data of the G-Bot while moving from ROOM 1 to ROOM 2. The data of 3 ultrasonic sensors, the data of the left and the right PWM were recorded in that table.

\subsubsection{Fuzzification for Case 2}

In the second calculation, an example is taken from the $7 \mathrm{~s}$ measurement. The data of each membership function in input fuzzy are based on Equation (6).

Table 6 Ultrasonic Distance Sensor Calculation Value

\begin{tabular}{|c|c|c|c|c|c|}
\hline \multirow{2}{*}{ No. } & \multicolumn{2}{|c|}{ Ultrasonic Distance Calculation Value } & \multicolumn{2}{|c|}{ PWM } \\
\cline { 2 - 6 } & S1 & S2 & S3 & left & Right \\
\hline \multirow{2}{*}{1} & $\begin{array}{c}\text { N } \\
0.53\end{array}$ & VF & VF & F & S \\
\hline \multirow{2}{*}{2} & $\begin{array}{c}\text { F } \\
0.46\end{array}$ & $\begin{array}{c}\text { VF } \\
1\end{array}$ & $\begin{array}{c}\text { VF } \\
1\end{array}$ & M & S \\
\hline
\end{tabular}

Table 7 Results of Ultrasonic Sensor Distance Defuzzification Calculations.

\begin{tabular}{|c|c|c|c|c|}
\hline $\begin{array}{c}\text { Least Value Fuzzy } \\
\text { Rule }(\alpha)\end{array}$ & $\begin{array}{c}\text { Left PWM } \\
(w \text { Left })\end{array}$ & $\begin{array}{c}\text { Right PWM } \\
(w \text { Right })\end{array}$ & w.aLeft & w.aRight \\
\hline 0.53 & 200 & 100 & 106 & 53 \\
\hline 0.46 & 150 & 100 & 69 & 46 \\
\hline$\Sigma \alpha=0.99$ & \multicolumn{2}{|c|}{ Total : } & 175 & 99 \\
\hline
\end{tabular}


From the experiments shown in Table 5 (for $\mathrm{t}=7$ ), it can be concluded that ultrasonic sensor distance parameters are, as follows: $\mathrm{S} 1=42, \mathrm{~S} 2=66$, and $\mathrm{S} 3=$ 74.

Therefore:

Ultrasonic sensor distance 42

$$
\begin{array}{lll}
\mathrm{N} & 35<\mathrm{X} \leq 50 & =\frac{x-50}{65-50}=\frac{42-50}{65-50}=0.53 \\
\mathrm{~F} & 35<\mathrm{X} \leq 50 & =\frac{x-35}{50-35}=\frac{42-35}{50-35}=0.46 \\
\mathrm{VF} & \mathrm{X}<50 & =0
\end{array}
$$

Ultrasonic sensor distance 66

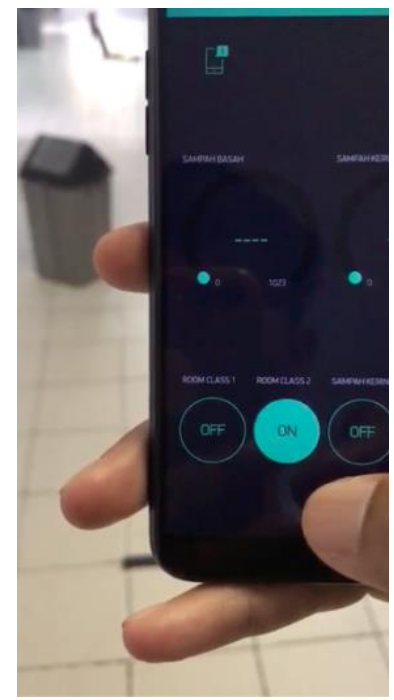

(a) ROOM 2 Button ON

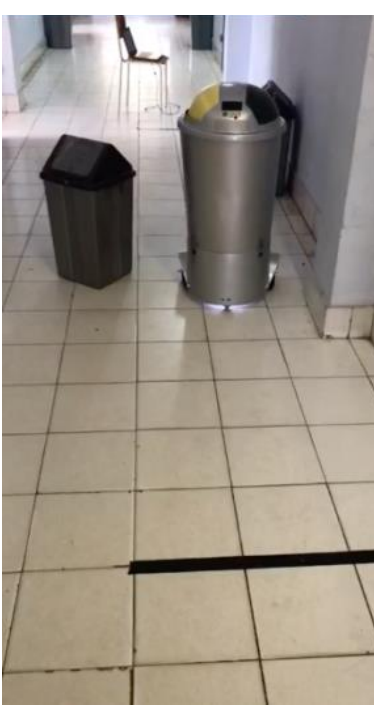

(e) $t=9 s$

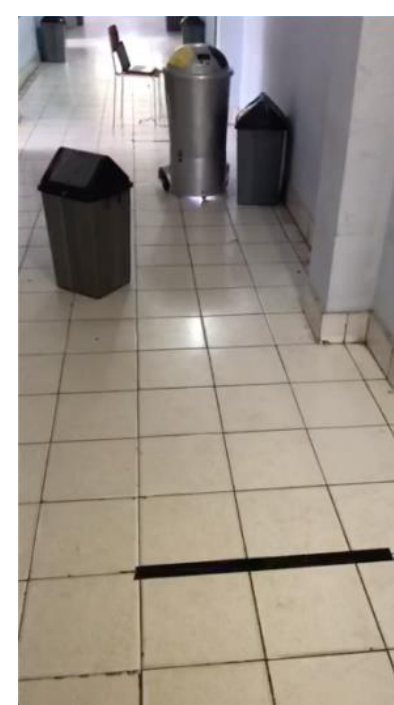

(b) $\mathrm{t}=3 \mathrm{~s}$

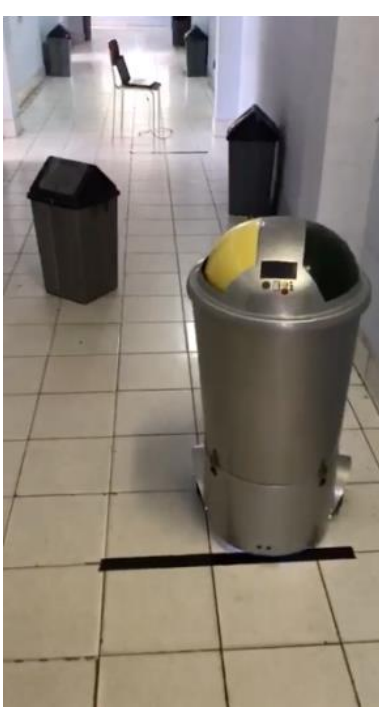

(f) $\mathrm{t}=13 \mathrm{~s}$

$\begin{array}{lll}\mathrm{N} & \mathrm{X}>50 & =0 \\ \mathrm{~F} & \mathrm{X}>65 & =0 \\ \text { VF } & \mathrm{X}>65 & =1\end{array}$

Ultrasonic sensor distance 74

$\begin{array}{lll}N & X>50 & =0 \\ F & X>65 & =0 \\ \text { VF } & X>65 & =1\end{array}$

Based on the above calculations, the equation is obtained, namely:

1. N VF VF

2. F VF VF

See Table 6 for the Detail of the ultrasonic distancee calculation vaue.

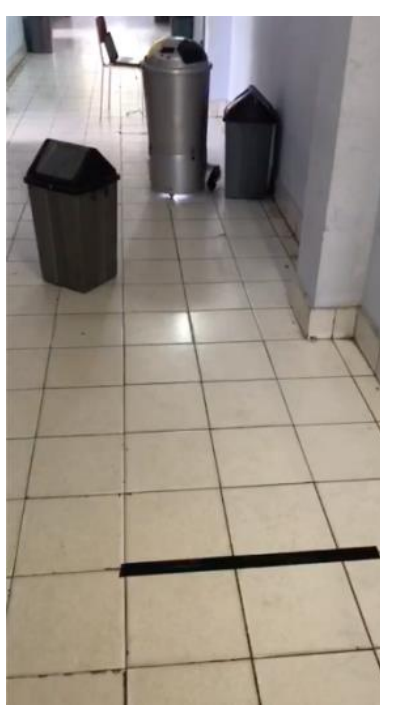

(c) $t=4 \mathrm{~s}$

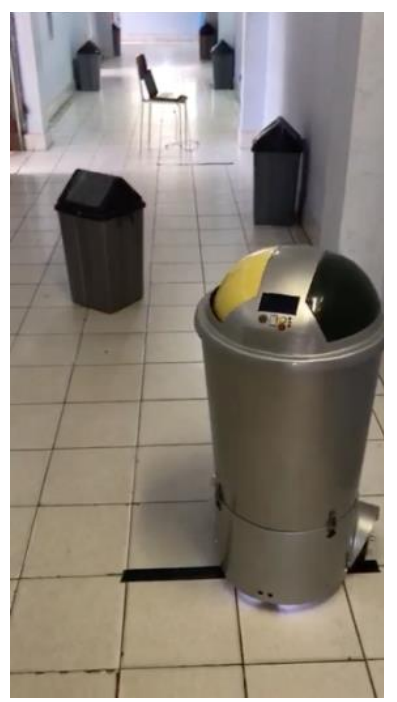

(g) $\mathrm{t}=14 \mathrm{~s}$

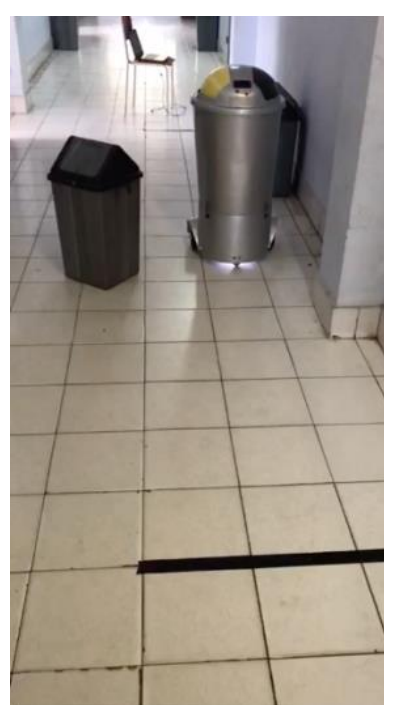

(d) $t=7 \mathrm{~s}$

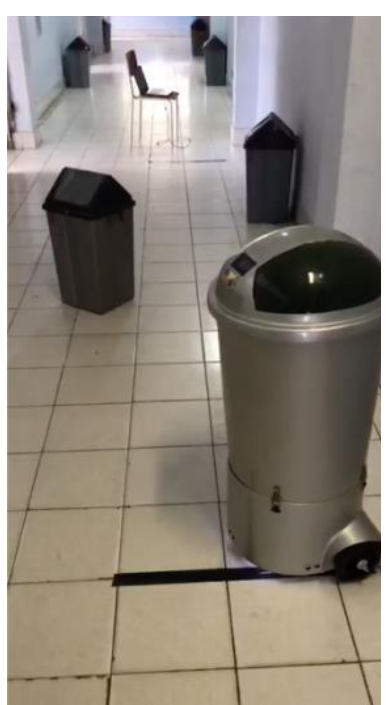

(h) $t=16 \mathrm{~s}$

Figure 10 Navigation of G-Bot in Case 2 


\subsubsection{Defuzzification for Case 2}

Table 7 shows the calculation of the ultrasonic sensor distance defuzzification. This data was obtained by calculating the value using the Equation (7). For $t=7 \mathrm{~s}$, it can be calculated that the rpm of left and the right motor 176.8 and 100 respectively

$\mathrm{Z}_{\text {Left }}=\frac{w . \alpha \text { Left }}{\sum \alpha}=\frac{175}{0.99}=176.8$

$\mathrm{Z}_{\text {Right }}=\frac{w \cdot \alpha \text { Right }}{\varepsilon \alpha}=\frac{99}{0.99}=100$

To test the result, compare the measurement and calculation value. The error of the left and right motor PWM can be calculated using Equation (9)

\section{Left Motor:}

error $=\left|\frac{176.8-179}{176.8}\right| \times 100 \%$

error $=\left|\frac{2.2}{176.8}\right| \times 100 \%$

error $=1.24 \%$

\section{Right Motor:}

error $=\left|\frac{100-97}{100}\right| \times 100 \%$

error $=\left|\frac{3}{100}\right| \times 100 \%$

error $=3 \%$

Thus, the output of the fuzzy logic (defuzzification) of the ultrasonic sensor distance is 176.8 (Left PWM) and 100 (Right PWM) with an error percentage of $1.24 \%$ for the left motor and $3 \%$ for the right motor. The results of these calculations can be compared to the experimental results at the 7th second of the right row, i.e., $t$ the results of the left PWM and the right PWM are almost the same with the experimental results.

\section{CONCLUSSION}

From the experimental testing, it can be concluded that the fuzzy logic system used in the robot motion control system can operate and can be applied in a navigation system. The G-Bot could hint the obstacles well using the fuzzy logic. The value generated from the readings of the $\mathrm{S} 1, \mathrm{~S} 2$, and S3 sensors greatly affected the movement of the robot with a set point of $35 \mathrm{~cm}$. From the results of the research and testing that has been done, the author provides some suggestions, such as: the use of a more responsive proximity sensor such as the PING sensor will be more helpful in robot maneuvers, and it is necessary to carry out continuous research and testing so that this G-Bot can be implemented properly.

\section{ACKNOWLEDGMENT}

The Authors thanks to Ministry of Education and Culture of Indonesia for the funding in this research. All thank also goes to all researchers in signal and processing Laboratory at State Polytechnic of Sriwijaya.

\section{REFERENCES}

[1] J. Monroy and J. Gonzalez-Jimenez, "Towards odor-sensitive mobile robots," Electron. Nose Technol. Adv. Mach. Olfaction, pp. 244-263, 2018, doi: 10.4018/978-1-5225-3862-2.ch012.

[2] J. G. Monroy and J. Gonzalez-jimenez, "Sensors and Actuators B : Chemical Gas classification in motion: An experimental analysis," Sensors Actuators B. Chem., vol. 240, pp. 1205-1215, 2017, doi: 10.1016/j.snb.2016.09.013.

[3] Nyayu Latifah Husni et al, "Garbage Box (GBox) Designing and Monitoring," ITC CSCC Conf., pp. 5-8, 2019.

[4] K. N. and V. N. Anukriti Jha, Anshuman Singh, Roshan Kerketta, Deepak Prasad, "Development of Autonomous Garbage Collector Robot," pringer Nat. Singapore Pte Ltd., vol. 24, pp. 689703, 2019, doi: 10.1007/978-981-10-6890-4.

[5] B. Yr, "Waste Workers and Occupational Health Risks," IJOSH, vol. 8, no. 2, pp. 1-3, 2018.

[6] Z. Wen et al., "Design, implementation, and evaluation of an Internet of Things (IoT) network system for restaurant food waste management," Waste Manag., vol. 73, pp. 26-38, 2018, doi: 10.1016/j.wasman.2017.11.054.

[7] J. Svub, P. Stasa, F. Benes, and J. Unucka, "RFID application in municipal waste management system," Inz. Miner., vol. 2017, no. 1, pp. 71-76, 2017.

[8] K. B. M.N. and A.-S. N.M., "On the Internet of Things, smart cities and the WHO Healthy Cities," Int. J. Health Geogr., vol. 13, no. 1, pp. 1-6, 2014, doi: 10.1186/1476-072X-13-10.

[9] D. Misra, G. Das, T. Chakrabortty, and D. Das, "An IoT-based waste management system monitored by cloud," J. Mater. Cycles Waste Manag., vol. 20, no. 3, pp. 1574-1582, 2018, doi: 10.1007/s10163-018-0720-y.

[10] N. L. Husni, Robi, E. Prihatini, Nurhaida, A. Silvia, and Firdaus, "Garbage Monitoring and Warning System," ICECOS 2019 - 3rd Int. Conf. Electr. Eng. Comput. Sci. Proceeding, pp. 171175, 2019, doi: 10.1109/ICECOS47637.2019.8984545. 
[11] C. Mayorga et al., "GABOT: Garbage autonomous collector for indoors at low cost," Proc. - 2019 Int. Conf. Mechatronics, Electron. Automot. Eng. ICMEAE 2019, pp. 56-61, 2019, doi: 10.1109/ICMEAE.2019.00018.

[12] N. L. Husni, S. Sitangsu, S. Rasyad, F. Damsi, and A. Silvia, "Real Time Garbage Bin Capacity Monitoring," Comput. Eng. Appl. J., vol. 9, no. 2, pp. 127-133, 2020.

[13] F. Cuevas and O. Castillo, "Design and Implementation of a Fuzzy Path Optimization System for Omnidirectional Autonomous Mobile Robot Control in Real-Time," pp. 241-252, 2018.

[14] O. Saleem, H. Hassan, A. Khan, and U. Javaid, "Adaptive Fuzzy-PD Tracking Controller for Optimal Visual-Servoing of Wheeled Mobile Robots," vol. 19, no. 3, pp. 58-68, 2017.

[15] A. S. Handayani, N. L. Husni, S. Nurmaini, and I. Yani, "Formation control design for real swarm robot using fuzzy logic," ICECOS 2017 Proceeding 2017 Int. Conf. Electr. Eng. Comput. Sci. Sustain. Cult. Herit. Towar. Smart Environ. Better Futur., pp. 77-82, 2017, doi: 10.1109/ICECOS.2017.8167171.

[16] A. S. Handayani, S. Nurmaini, I. Yani, and N. L.
Husni, "Analysis on swarm robot coordination using fuzzy logic," Indones. J. Electr. Eng. Comput. Sci., vol. 13, no. 1, pp. 48-57, 2019, doi: 10.11591/ijeecs.v13.i1.pp48-57.

[17] N. L. Husni, A. Silvia, S. Nurmaini, and R. Yani, "Optimal Gas Sensors Arrangement in Odor Searching Robot," Proc. 2018 Int. Conf. Electr. Eng. Comput. Sci. ICECOS 2018, vol. 17, pp. 111-116, 2019 , 10.1109/ICECOS.2018.8605236.

[18] N. L. Husni, A. S. Handayani, S. Nurmaini, and I. Yani, "Odor localization using gas sensor for mobile robot," Int. Conf. Electr. Eng. Comput. Sci. Informatics, vol. 4, no. September, pp. 584589, 2017, doi: 10.11591/eecsi.4.1087.

[19] N. L. Husni, N. Lukita, M. Anisah, A. S. Handayani, S. Nurmaini, and I. Yani, "Enhancement of Navigation Systems of Mobile Robots in Gas Leakage Searching," J. Phys. Conf. Ser., vol. 1167, no. 1, 2019, doi: 10.1088/1742-6596/1167/1/012005.

[20] N. L. Husni and A. S. Handayani, "Odor Localization using Gas Sensor for Mobile Robot." 